\title{
New Epoch for Arterial Stiffness Measurement in the Clinic
}

\author{
Jeong Bae Park ${ }^{a}$ Kazuomi Kario ${ }^{b}$ \\ a Division of Cardiology, Department of Medicine, Cheil General Hospital, Dankook \\ University College of Medicine, Seoul, South Korea; ${ }^{b}$ Division of Cardiovascular Medicine, \\ Department of Medicine, Jichi Medical University School of Medicine, Tochigi, Japan
}

Age changes the vascular structure and function in the normal population, but it has a different effect on wave reflection and pulse wave transmission [1,2]. Many longitudinal studies have found that arterial stiffness measurement among hypertensive patients, diabetic patients, healthy elderly, and people with high cardiovascular (CV) risk will better evaluate arterial health and predict CV events. European guidance for arterial stiffness measurements described in the European consensus document (2006) [3], the publication of the international reference standards (2010) [4] and the American Heart Association (AHA) scientific statement on arterial stiffness measurements in the USA (2015) have standardized arterial measurement [5], and thus arterial stiffness measurements have been more widely used in clinical settings. The question now is which measurement of arterial stiffness is more accurate to precisely predict future CV events. Most of the guidelines designated aortic pulse wave velocity (PWV; carotid-femoral PWV) as the golden standard (especially in the Western society) [6], but in Japan, where arterial stiffness is the most widely used and where they achieved a great amount of data on brachial-ankle PWV and cardio-ankle vascular index (CAVI) [7], studies found a relationship between organ damage and future CV events. Townsend's [8] brief review on recommendations and standardization of PWV measurements also justified its clinical and scholarly use.

The articles from Korea [9] and Thailand [10] describe CV risk factors and outcomes using the CAVI. The modified RAMA-EGAT score from Thailand combined the conventional risk prevention model with arterial function. It would be helpful to have age- and genderstratified values of the CAVI in healthy Koreans and in high-risk patients of a metabolically risky condition. The study from Thailand developed its own risk prediction model called the RAMA-EGAT score, which proved to be accurate in the prediction of CV risk in patients with intermediate atherosclerotic risk factors in the Thai population [11], and a further modification of the RAMA-EGAT score with the CAVI enhanced its accuracy. Now they are conducting a long-term prognosis study in patients with diverse risk conditions. The Framingham-CAVI 
score from Japan [12] is very impressive as well. In many cohort studies, further estimation of arterial structure and function in addition to traditional risk factors have been found to increase the prediction rate of future CV risk, and the fact that the CAVI is widely used in Thailand and Japan indicates that the analysis of arterial structure and function is becoming normal in clinical settings. Based on the studies conducted in Asia reporting on clinical experience and data on the CAVI, the CAVI is expected to be widely used in Europe or the US as well, and further consensus and clinical experience in Western societies will increase its applicability. We believe that the CAVI to predict CV events in Japan (CAVI-J), which examines patients with common high-risk factors such as diabetes mellitus, metabolic syndrome, highrisk hypertension, chronic kidney disease, history of ischemic heart and brain disease for 5 years, will give us further information. In that sense, the mini-reviews of this issue will be a good start.

Jeong Bae Park, Seoul Kazuomi Kario, Tochigi

\section{Disclosure Statement}

The authors declare that no conflicts of interest exist.

\section{References}

1 McEniery CM, Yasmin, Hall IR, Qasem A, Wilkinson IB, Cockcroft JR: Normal vascular aging: differential effects on wave reflection and aortic pulse wave velocity: the Anglo-Cardiff Collaborative Trial (ACCT). J Am Coll Cardiol 2005; 46:1753-1760.

2 Choi SY, Oh BH, Bae Park J, Choi DJ, Rhee MY, Park S: Age-associated increase in arterial stiffness measured according to the cardio-ankle vascular index without blood pressure changes in healthy adults. J Atheroscler Thromb 2013;20:911-923.

3 Laurent S, Cockcroft J, Van Bortel L, Boutouyrie P, Giannattasio C, Hayoz D, Pannier B, Vlachopoulos C, Wilkinson I, Struijker-Boudier H: Expert consensus document on arterial stiffness: methodological issues and clinical applications. Eur Heart J 2006;27:2588-2605.

4 Reference Values for Arterial Stiffness Collaboration: Determinants of pulse wave velocity in healthy people and in the presence of cardiovascular risk factors: 'establishing normal and reference values'. Eur Heart J 2010;31:2338-2350.

5 Townsend RR, Wilkinson IB, Schiffrin EL, Avolio AP, Chirinos JA, Cockcroft JR, Heffernan KS, Lakatta EG, McEniery CM, Mitchell GF, Najjar SS, Nichols WW, Urbina EM, Weber T: Recommendations for improving and standardizing vascular research on arterial stiffness: a scientific statement from the American Heart Association. Hypertension 2015;66:698-722.

6 Van Bortel LM, Laurent S, Boutouyrie P, Chowienczyk P, Cruickshank JK, De Backer T, Filipovsky J, Huybrechts S, Mattace-Raso FU, Protogerou AD, Schillaci G, Segers P, Vermeersch S, Weber T: Expert consensus document on the measurement of aortic stiffness in daily practice using carotid-femoral pulse wave velocity. J Hypertens 2012;30:445-448.

7 Shirai K, Utino J, Otsuka K, Takata M: A novel blood pressure-independent arterial wall stiffness parameter; cardio-ankle vascular index (CAVI). J Atheroscler Thromb 2006;13:101-107.

8 Townsend RR: Arterial stiffness: recommendations and standardization. Pulse 2016;4(suppl 1):3-7.

9 Choi SY: Clinical application of the cardio-ankle vascular index in asymptomatic healthy Koreans. Pulse 2016; 4(suppl 1):17-20.

10 Yingchoncharoen T, Sritara P: Cardio-ankle vascular index in a Thai population. Pulse 2016;4(suppl 1):8-10.

11 Yingchoncharoen T, Limpijankit T, Jongjirasiri S, Laothamatas J, Yamwong S, Sritara P: Arterial stiffness contributes to coronary artery disease risk prediction beyond the traditional risk score (RAMA-EGAT score). Heart Asia 2012;4:77-82.

12 Miyoshi T, Ito H, Horinaka S, Shirai K, Higaki J, Orimio H: Protocol for evaluating the cardio-ankle vascular index to predict cardiovascular events in Japan: a prospective multicenter cohort study. Pulse 2016;4(suppl 1): $11-16$. 